\title{
Convenient asymmetric synthesis of 1,3,4,6-tetrasubstituted 2,5-diketopiperazines
}

\author{
Myung-su Lee, Jinho Baek, Eunjee Youk, Yongtae Kim, and Yong Sun Park* \\ Department of Chemistry, Konkuk University, Seoul 05029, Korea \\ E-mail:parkyong@,konkuk.ac.kr
}

DOI: http://dx.doi.org/10.3998/ark.5550190.p009.532

\begin{abstract}
A general and efficient method was developed for the asymmetric synthesis of 1,3,4,6tetrasubstituted 2,5-diketopiperazines. The asymmetric nucleophilic substitution of L-amino acid-derived $\alpha$-bromo tertiary amides with aliphatic primary amines followed by spontaneous cyclization provides a rapid access to diverse 1,3,4,6-tetrasubstituted-2,5-diketopiperazines with high diastereoselectivities of up to 99:1 dr.
\end{abstract}

Keywords: Nucleophilic substitution, amino acid, chirality, peptidomimetics, asymmetric synthesis

\section{Introduction}

2,5-Diketopiperazines with four substituents at the 1,3,4 and 6 positions are interesting scaffolds for drug design and peptide chemistry, because the substituents of the rigid cyclic dipeptide can be arranged in a well-defined spatial manner. ${ }^{1-2}$ Among them, special attention has been paid to unsymmetrical tetra-substituted diketopiperazines which can provide a unique opportunity to design suitable drug candidates. ${ }^{3-6}$ (Figure 1) Thus, asymmetric synthetic methods for unsymmetrical tetrasubstituted diketopiperazines are highly desirable. However, the synthetic methods involving naturally occurring L-amino acids are often limited by the diversity of the substituents. Furthermore, the syntheses of tetrasubstituted diketopiperazines require either multistep sequences or harsh conditions and they are inefficient for asymmetric synthesis. ${ }^{7-13}$ As an extension of our previous work on the asymmetric preparation of 3,4,6-trisubstituted 2,5diketopiperazines, ${ }^{14}$ we herein report a mild and versatile synthetic method for the asymmetric synthesis of 1,3,4,6-tetrasubstituted 2,5-diketopiperazines via one-pot substitution-cyclization of $\alpha$-bromo tertiary acetamides (Figure 1 ). 

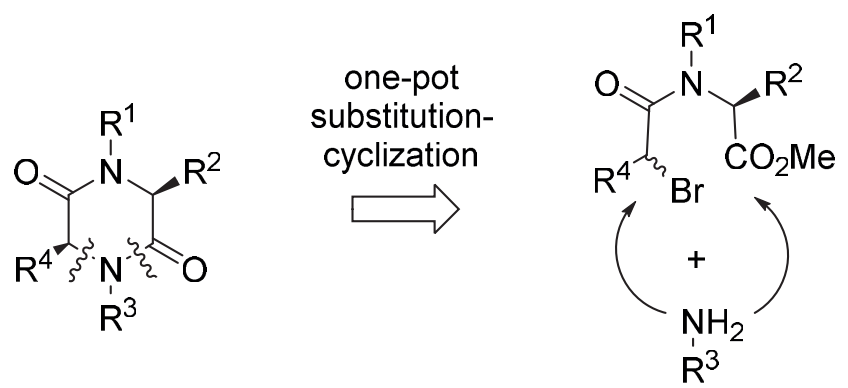

Figure 1. Retrosynthesis of unsymmetrical 1,3,4,6-tetrasubstituted 2,5-diketopiperazines $\left(\mathrm{R}^{1} \neq \mathrm{R}^{3}\right.$ and $\left.\mathrm{R}^{2} \neq \mathrm{R}^{4}\right)$.

\section{Results and Discussion}

We previously reported that the dynamic kinetic resolution of L-amino acid-derived $\alpha$-bromo tertiary acetamides in nucleophilic substitution with $p$-anisidine affords highly diastereoenriched dipeptide analogs and subsequent deprotection-cyclization process gives 3,4,6-trisubstituted 2,5diketopiperazines. ${ }^{14}$ In our efforts to extend the synthetic method to the asymmetric synthesis of tetrasubstituted 2,5-diketopiperazines, we have found a simple and convenient synthetic method involving the substitution and spontaneous cyclization with primary aliphatic amines.

In contrast to the reaction of $\alpha$-bromo tertiary acetamide 1 with $p$-anisidine, the substitution of 1 with benzylamine spontaneously afforded a cyclized product 2 under the same conditions (Table 1, entries 1 and 2). The tetrasubstituted 2,5-diketopiperazine was produced in $88 \%$ yield and with 98:2 dr after $48 \mathrm{~h}$ stirring with DIEA and TBAI in $\mathrm{CH}_{3} \mathrm{CN}$ by the dynamic kinetic resolution of $\alpha$-bromo tertiary acetamide 1 (50:50 dr). ${ }^{15-20} \mathrm{Next}$, several primary amine nucleophiles were screened as shown in Table 1; most of the primary amines successfully afforded tetrasubstituted 2,5-diketopiperazines. The reactions of $\mathbf{1}$ with $n$-propylamine, $n$ butylamine, iso-butylamine, 2-phenethylamine and methylamine (2.0 M solution in THF) afforded diketopiperazines 3-7 in yields ranging from 51\% to $88 \%$ and with comparably high stereoselectivities, as shown in entries 3-7. More sterically demanding isopropylamine afforded a substitution product, but no cyclization occurred under the same reaction conditions (entry 8). The synthetic method was extended to functionalized amine nucleophiles to obtain diverse diketopiperazines with additional functionalities. The reactions of $\mathbf{1}$ with amines containing methoxycarbonylmethyl, $N$-Boc-aminoethyl, indol-3-ylethyl and $p$-methoxybenzyl groups also afforded highly diastereoenriched diketopiperazines 8-11 with 96:4, 96:4, 95:5 and 96:4 drs, respectively (entries 9-12). 
Table 1. Synthesis of tetrasubstituted 2,5-diketopiperazines using alkyl amines<smiles>COC(=O)C(C)N(Cc1ccccc1)C(=O)C(Br)c1ccccc1</smiles>

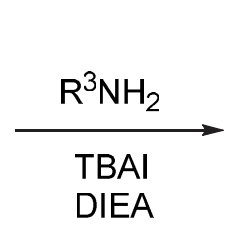

1<smiles>[R]N1C(=O)C(C)N(Cc2ccccc2)C(=O)C1c1ccccc1</smiles>

\begin{tabular}{lllll}
\hline Entry $^{\mathrm{a}}$ & $\mathrm{R}^{3}$ & $\mathrm{Dr}(\alpha \mathrm{S}: \alpha R)^{\mathrm{b}}$ & Product & Yield (\%) $^{\mathrm{c}}$ \\
\hline 1 & $p-\mathrm{MeOC}_{6} \mathrm{H}_{4}$ & - & - & - \\
2 & $\mathrm{PhCH}_{2}$ & $98: 2$ & $\mathbf{2}$ & 88 \\
3 & $\mathrm{CH}_{3} \mathrm{CH}_{2} \mathrm{CH}_{2}$ & $95: 5$ & $\mathbf{3}$ & 88 \\
4 & $\mathrm{CH}_{3} \mathrm{CH}_{2} \mathrm{CH}_{2} \mathrm{CH}_{2}$ & $95: 5$ & 4 & 72 \\
5 & $\left(\mathrm{CH}_{3}\right)_{2} \mathrm{CHCH}_{2}$ & $96: 4$ & $\mathbf{5}$ & 66 \\
6 & $\mathrm{PhCH}_{2} \mathrm{CH}_{2}$ & $94: 6$ & $\mathbf{6}$ & 75 \\
7 & $\mathrm{CH}_{3}$ & $96: 4$ & 7 & 51 \\
8 & $\left(\mathrm{CH}_{3}\right)_{2} \mathrm{CH}_{9}$ & - & - & - \\
9 & $\mathrm{MeO}_{2} \mathrm{CCH}_{2}$ & $96: 4$ & $\mathbf{8}$ & 76 \\
10 & $\mathrm{BocNHCH}_{2} \mathrm{CH}_{2}$ & $96: 4$ & $\mathbf{9}$ & 60 \\
11 & $($ indol-3-yl $) \mathrm{CH}_{2} \mathrm{CH}_{2}$ & $95: 5$ & $\mathbf{1 0}$ & 83 \\
12 & $p-\mathrm{MeOC}_{6} \mathrm{H}_{4} \mathrm{CH}_{2}$ & $96: 4$ & $\mathbf{1 1}$ & 77 \\
\hline
\end{tabular}

${ }^{a}$ All the reactions were carried out in $\mathrm{CH}_{3} \mathrm{CN}$ at $\mathrm{rt}$ for $48 \mathrm{~h}$. ${ }^{\mathrm{b}}$ The drs were determined by the ${ }^{1} \mathrm{H}$ NMR spectra of the reaction mixtures. ${ }^{\mathrm{c}}$ Isolated yields.

To establish the generality of the synthetic methodology, the substrate scope of the stereoselective diketopiperazine formation was investigated using four different L-amino acid precursors (Table 2). The substitution reactions of $N$-benzyl substituted $\alpha$-bromo acetamides 12 15 derived from L-phenylalanine, L-leucine, L-aspartic acid, and L-glutamic acid were studied as shown in entries 1-4. The reaction of L-Phe-derived $\alpha$-bromo acetamide 12 with 2phenylethylamine, TBAI and DIEA afforded diketopiperazine 20 with 96:4 dr (entry 1). Almost the same selectivities were observed with L-Leu, L-Asp, and L-Glu-derived $\alpha$-bromo acetamides 13-15 (entries 2-4). Next, the effect of $\mathrm{R}^{4}$ substituents on the stereoselectivity of the substitution was studied (entries 5-8). Two aromatic and two aliphatic substituents $\left(\mathrm{R}^{2}\right)$ such as $p$ chlorophenyl, $p$-bromophenyl, methyl and ethyl groups were investigated. The reactions of $\alpha$ bromo acetamides 16 and 17 with 2-phenylethylamine, TBAI and DIEA afforded the corresponding products with a slightly lower drs of 92:8 and 93:7, respectively. The reactions of $\alpha$-bromo acetamides 18 and 19 bearing aliphatic $\mathrm{R}^{4}$ substituents showed much lower selectivities of 87:13 and 90:10 drs, respectively, under identical reaction conditions. 
Table 2. Synthesis of tetrasubstituted 2,5-diketopiperazines with different $R^{2}$ and $R^{4}$ substituents<smiles>[R]C(Br)C(=O)N(Cc1ccccc1)C([R])C(=O)OC</smiles><smiles>NCCc1ccccc1</smiles>

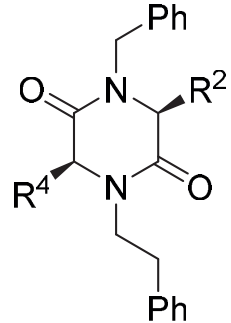

\begin{tabular}{llllllc}
\hline Entry & Reactant & $\mathrm{R}^{2}$ & $\mathrm{R}^{4}$ & $\begin{array}{l}\mathrm{Dr} \\
(\alpha S: \alpha R)^{\mathrm{b}}\end{array}$ & Product & $\begin{array}{c}\text { Yield } \\
(\%)^{\mathrm{c}}\end{array}$ \\
\hline 1 & $\mathbf{1 2}$ & $\mathrm{PhCH}_{2}$ & $\mathrm{Ph}$ & $96: 4$ & $\mathbf{2 0}$ & 56 \\
2 & $\mathbf{1 3}$ & $\left(\mathrm{CH}_{3}\right)_{2} \mathrm{CHCH}_{2}$ & $\mathrm{Ph}$ & $97: 3$ & $\mathbf{2 1}$ & 70 \\
3 & $\mathbf{1 4}$ & $\mathrm{MeO}_{2} \mathrm{CCH}_{2}$ & $\mathrm{Ph}$ & $97: 3$ & $\mathbf{2 2}$ & 71 \\
4 & $\mathbf{1 5}$ & $\mathrm{MeO}_{2} \mathrm{CCH}_{2} \mathrm{CH}_{2}$ & $\mathrm{Ph}$ & $94: 6$ & $\mathbf{2 3}$ & 81 \\
5 & $\mathbf{1 6}$ & $\left(\mathrm{CH}_{3}\right)_{2} \mathrm{CHCH}_{2}$ & $p-\mathrm{ClC}_{6} \mathrm{H}_{4}$ & $92: 8$ & $\mathbf{2 4}$ & 48 \\
6 & $\mathbf{1 7}$ & $\left(\mathrm{CH}_{3}\right)_{2} \mathrm{CHCH}_{2}$ & $p-\mathrm{BrC}_{6} \mathrm{H}_{4}$ & $93: 7$ & $\mathbf{2 5}$ & 51 \\
7 & $\mathbf{1 8}$ & $\left(\mathrm{CH}_{3}\right)_{2} \mathrm{CHCH}_{2}$ & $\mathrm{CH}_{3}$ & $87: 13$ & $\mathbf{2 6}$ & 89 \\
8 & $\mathbf{1 9}$ & $\left(\mathrm{CH}_{3}\right)_{2} \mathrm{CHCH}_{2}$ & $\mathrm{CH}_{3} \mathrm{CH}_{2}$ & $90: 10$ & $\mathbf{2 7}$ & 75 \\
\hline
\end{tabular}

${ }^{a}$ All the reactions were carried out in $\mathrm{CH}_{3} \mathrm{CN}$ at $\mathrm{rt}$ for $48 \mathrm{~h} .{ }^{\mathrm{b}}$ The drs were determined by the $\mathrm{H}$ NMR spectra of the reaction mixtures. ${ }^{c}$ Isolated yields.

Encouraged by the observed high stereoselectivity in the reaction of $N$-benzyl substituted $\alpha$ bromo acetamides with primary amines, $N$-( $p$-methoxybenzyl) substituted $\alpha$-bromo acetamides 28-30 were investigated. $N$-( $p$-methoxybenzyl) group can be used for the temporary protection of the tertiary amide bond, affording an $N$-unsubstituted 2,5-diketopiperazine. ${ }^{21-22}$ The reactions of L-leucine-derived 28 and L-alanine-derived 29 with benzylamine afforded $N$-( $p$-methoxybenzyl) substituted diketopiperazines $\mathbf{3 5}$ and $\mathbf{3 6}$ in good yields and with excellent drs of 98:2 and 99:1, respectively (entries 1 and 2). The reactions of 29 with $n$-propylamine and $p$ methoxybenzylamine afforded highly diastereoenriched 37 and 38, respectively (entries 3 and 4). We were pleased to observe that $\mathbf{3 0}$ bearing an aliphatic methyl substituent $\left(\mathrm{R}^{4}\right)$ also afforded a tetrasubstituted 2,5-diketopiperazine 39 with 99:1 dr. We confirmed the cis configuration of diketopiperazines 38 and 39 by comparing their NMR spectra with those of previously reported compounds. ${ }^{23-26}$ The reactions of $N$-(trans-cinnamyl) substituted $\alpha$-bromo acetamides 31 and 32 produced highly functionalized diketopiperazines $\mathbf{4 0}$ and $\mathbf{4 1}$ with stereoselectivities of 99:1 and 92:8 dr, respectively. Finally, this methodology was found to be efficient for the synthesis of Lproline-derived diketopiperazines with high stereoselectivities and good yields as shown in entries 8-10. The reactions of L-proline-derived $\alpha$-bromo phenylacetamide 33 with 2- 
phenylethylamine and $p$-methoxybenzylamine afforded the corresponding transdiketopiperazines $\mathbf{4 2}$ and $\mathbf{4 3}$ with 93:7 and 96:4 drs, respectively. However, a much lower stereoselectivity of 75:25 dr was observed in the reactions of L-proline-derived $\alpha$-bromo propionamide 34 bearing an aliphatic $\mathrm{R}^{4}$ substituent, affording trans-diketopiperazine 44 in $76 \%$ yield (entry 10). ${ }^{25-26}$

Table 3. Stereoselective synthesis of 1,3,4,6-tetrasubstituted 2,5-diketopiperazines<smiles>[R]C(Br)C(=O)N([R])C([R])C([R])Br</smiles>

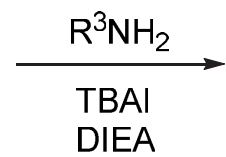<smiles>[R]C1=NC(=O)C([R])N([R])C1=O</smiles>

\begin{tabular}{|c|c|c|c|c|c|c|c|}
\hline Entry $^{\mathrm{a}}$ & $\mathrm{R}^{1}$ & $\mathrm{R}^{2}$ & $\mathrm{R}^{3}$ & $\mathrm{R}^{4}$ & $\begin{array}{l}\text { Dr } \\
(\alpha S: \alpha R)^{\mathrm{b}}\end{array}$ & Product & $\begin{array}{l}\text { Yield } \\
(\%)^{\mathrm{c}}\end{array}$ \\
\hline $1(\mathbf{2 8})$ & $p-\mathrm{MeOC}_{6} \mathrm{H}_{4} \mathrm{CH}_{2}$ & $\left(\mathrm{CH}_{3}\right)_{2} \mathrm{CHCH}_{2}$ & $\mathrm{PhCH}_{2}$ & $\mathrm{Ph}$ & $98: 2$ & 35 & 70 \\
\hline $2(\mathbf{2 9})$ & $p-\mathrm{MeOC}_{6} \mathrm{H}_{4} \mathrm{CH}_{2}$ & $\mathrm{CH}_{3}$ & $\mathrm{PhCH}_{2}$ & $\mathrm{Ph}$ & $99: 1$ & 36 & 73 \\
\hline $3(29)$ & $p-\mathrm{MeOC}_{6} \mathrm{H}_{4} \mathrm{CH}_{2}$ & $\mathrm{CH}_{3}$ & $\mathrm{CH}_{3} \mathrm{CH}_{2} \mathrm{CH}_{2}$ & $\mathrm{Ph}$ & $99: 1$ & 37 & 80 \\
\hline $4(29)$ & $p-\mathrm{MeOC}_{6} \mathrm{H}_{4} \mathrm{CH}_{2}$ & $\mathrm{CH}_{3}$ & $p-\mathrm{MeOC}_{6} \mathrm{H}_{4} \mathrm{CH}_{2}$ & $\mathrm{Ph}$ & $96: 4$ & 38 & 68 \\
\hline $5(30)$ & $p-\mathrm{MeOC}_{6} \mathrm{H}_{4} \mathrm{CH}_{2}$ & $\mathrm{CH}_{3}$ & $p-\mathrm{MeOC}_{6} \mathrm{H}_{4} \mathrm{CH}_{2}$ & $\mathrm{CH}_{3}$ & $99: 1$ & 39 & 60 \\
\hline $6(31)$ & $\mathrm{PhCH}=\mathrm{CHCH}_{2}$ & $\left(\mathrm{CH}_{3}\right)_{2} \mathrm{CHCH}_{2}$ & $\mathrm{PhCH}_{2} \mathrm{CH}_{2}$ & $\mathrm{Ph}$ & $99: 1$ & 40 & 56 \\
\hline $7(32)$ & $\mathrm{PhCH}=\mathrm{CHCH}_{2}$ & $\mathrm{CH}_{3}$ & $p-\mathrm{MeOC}_{6} \mathrm{H}_{4} \mathrm{CH}_{2}$ & $\mathrm{Ph}$ & $92: 8$ & 41 & 77 \\
\hline $8(\mathbf{3 3})$ & \multicolumn{2}{|c|}{$-\mathrm{CH}_{2} \mathrm{CH}_{2} \mathrm{CH}_{2-}$} & $\mathrm{PhCH}_{2} \mathrm{CH}_{2}$ & $\mathrm{Ph}$ & $7: 93$ & 42 & 66 \\
\hline $9(\mathbf{3 3})$ & \multicolumn{2}{|c|}{$-\mathrm{CH}_{2} \mathrm{CH}_{2} \mathrm{CH}_{2-}$} & $p-\mathrm{MeOC}_{6} \mathrm{H}_{4} \mathrm{CH}_{2}$ & $\mathrm{Ph}$ & $4: 96$ & 43 & 87 \\
\hline $10(34)$ & \multicolumn{2}{|c|}{$-\mathrm{CH}_{2} \mathrm{CH}_{2} \mathrm{CH}_{2-}$} & $p-\mathrm{MeOC}_{6} \mathrm{H}_{4} \mathrm{CH}_{2}$ & $\mathrm{CH}_{3}$ & $25: 75$ & 44 & 76 \\
\hline
\end{tabular}

${ }^{\text {a }}$ All the reactions were carried out in $\mathrm{CH}_{3} \mathrm{CN}$ at $\mathrm{rt}$ for $48 \mathrm{~h}$. Numbers in parentheses after entry numbers indicate reactant numbers. ${ }^{b}$ The drs were determined by the H NMR spectra of the reaction mixtures. ${ }^{\mathrm{c}}$ Isolated yields.

\section{Conclusions}

A mild one-pot synthetic method was developed for the asymmetric synthesis of 1,3,4,5tetrasubstituted 2,5-diketopiperazines. The highly stereoselective process includes the dynamic kinetic resolution of $\alpha$-bromo tertiary amides in asymmetric substitution with a primary aliphatic amine as the key reaction. The following spontaneous cyclization provides 1,3,4,6tetrasubstituted 2,5-diketopiperazines. The asymmetric synthetic methodology provides a rapid 
access to diverse highly functionalized diketopiperazines. By the introduction of appropriate substituents in four defined positions, the diketopiperazines could be used as scaffolds for further functionalization in the asymmetric synthesis of complex target molecules.

\section{Experimental Section}

General. All reactions were performed in oven-dried glassware under nitrogen atmosphere. All chemicals were obtained from commercial sources and were used as received. Analytical thin layer chromatography (TLC) was performed on silica gel plates with QF-254 indicator and TLC visualization was carried out with UV-light. Flash column chromatography was performed with 230-400 mesh silica gel. ${ }^{1} \mathrm{H}$ and ${ }^{13} \mathrm{C}$ NMR spectra were acquired on Bruker $\left(400 \mathrm{MHz}{ }^{1} \mathrm{H}, 100.6\right.$ $\left.\mathrm{MHz}{ }^{13} \mathrm{C}\right)$ spectrometer using chloroform- $d\left(\mathrm{CDCl}_{3}\right)$ as the internal standard. Chemical shifts $(\delta)$ are reported in ppm relative to chloroform- $d\left(7.26 \mathrm{ppm}{ }^{1} \mathrm{H}, 77.07 \mathrm{ppm}{ }^{13} \mathrm{C}\right)$. Multiplicities are indicated by: $\mathrm{s}$ (singlet), d (doublet), $\mathrm{t}$ (triplet), q (quartet) and br (broad). Coupling constants $(J)$ are reported in Hz. HRMS spectra were measured on a JEOL JMS-700 by using ESI or FAB method.

General procedure for the preparation of 1,3,4,6-tetrasubstituted 2,5-diketopiperazines. To a solution of L-amino acid-derived $(\alpha R S)$ - $\alpha$-bromo tertiary acetamide $(1.0 \mathrm{mmol})$ in dry $\mathrm{CH}_{3} \mathrm{CN}$ $(\approx 0.1 \mathrm{M}$ of substrate) at room temperature was added an amine (1.2 equiv), TBAI (1.0 equiv) and DIEA (1.0 equiv). The resulting reaction mixture was stirred at room temperature for $48 \mathrm{~h}$ before the solvent was evaporated in vacuo. The resulting residue was purified by column chromatography on silica gel to give 1,3,4,6-tetrasubstituted 2,5-diketopiperazines. The purity ( $>95 \%$ ) of products was estimated by NMR spectroscopy.

(3S,6S)-1,4-Dibenzyl-6-methyl-3-phenylpiperazine-2,5-dione (2) A colorless oil was obtained in $88 \%$ yield. ${ }^{1} \mathrm{H}$ NMR $\left(\mathrm{CDCl}_{3}, 400 \mathrm{MHz}\right) 7.45-7.14(\mathrm{~m}, 15 \mathrm{H}), 5.59(\mathrm{~d}, J 14.8 \mathrm{~Hz}, 1 \mathrm{H}), 5.12(\mathrm{~d}$, $J 14.8 \mathrm{~Hz}, 1 \mathrm{H}), 5.10$ (s, 1H), 4.07 (q, $J 6.8 \mathrm{~Hz}, 1 \mathrm{H}), 3.96(\mathrm{~d}, J 14.8 \mathrm{~Hz}, 1 \mathrm{H}), 3.59$ (d, $J 14.4 \mathrm{~Hz}$, $1 \mathrm{H}), 1.45(\mathrm{~d}, J 7.2 \mathrm{~Hz}, 3 \mathrm{H}) ;{ }^{13} \mathrm{C} \mathrm{NMR}\left(\mathrm{CDCl}_{3}, 100 \mathrm{MHz}\right) 168.0,164.8,135.7,135.6,135.2$, 135.1, 129.1, 129.0, 128.9, 128.7, 128.4, 128.2, 128.0, 126.6, 62.4, 55.2, 47.6, 47.4, 19.1; HRMS calcd for $\mathrm{C}_{25} \mathrm{H}_{24} \mathrm{~N}_{2} \mathrm{O}_{2}\left(\mathrm{M}^{+}\right)$: 384.1838. Found 384.1839.

(3S,6S)-1-Benzyl-6-methyl-3-phenyl-4-n-propylpiperazine-2,5-dione (3) A colorless oil was obtained in $88 \%$ yield. ${ }^{1} \mathrm{H}$ NMR $\left(\mathrm{CDCl}_{3}, 400 \mathrm{MHz}\right) 7.46-7.20(\mathrm{~m}, 10 \mathrm{H}), 5.23(\mathrm{~s}, 1 \mathrm{H}), 5.17(\mathrm{~d}, J$ $14.8 \mathrm{~Hz}, 1 \mathrm{H}), 4.09-3.93(\mathrm{~m}, 3 \mathrm{H}), 2.66-2.59(\mathrm{~m}, 1 \mathrm{H}), 1.64-1.55(\mathrm{~m}, 2 \mathrm{H}), 1.37$ (d, J 6.8 Hz, 3H), $0.88(\mathrm{t}, J 7.6 \mathrm{~Hz}, 3 \mathrm{H}) ;{ }^{13} \mathrm{C} \mathrm{NMR}\left(\mathrm{CDCl}_{3}, 100 \mathrm{MHz}\right) 168.0,165.0,135.6,135.5,129.0,128.9$, $128.6,128.1,128.0,126.4,63.4,55.1,47.3,47.0,20.4,18.7,11.2$; HRMS calcd for $\mathrm{C}_{21} \mathrm{H}_{24} \mathrm{~N}_{2} \mathrm{O}_{2}$ $\left(\mathrm{M}^{+}\right)$: 336.1838. Found 336.1836.

(3S,6S)-1-Benzyl-4-n-butyl-6-methyl-3-phenylpiperazine-2,5-dione (4) A colorless oil was obtained in $72 \%$ yield. ${ }^{1} \mathrm{H}$ NMR $\left(\mathrm{CDCl}_{3}, 400 \mathrm{MHz}\right) 7.46-7.20(\mathrm{~m}, 10 \mathrm{H}), 5.23(\mathrm{~s}, 1 \mathrm{H}), 5.15(\mathrm{~d}, J$ $15.2 \mathrm{~Hz}, 1 \mathrm{H}), 4.14-3.94(\mathrm{~m}, 3 \mathrm{H}), 2.67-2.60(\mathrm{~m}, 1 \mathrm{H}), 1.58-1.54(\mathrm{~m}, 2 \mathrm{H}), 1.36(\mathrm{~d}, J 6.8 \mathrm{~Hz}, 3 \mathrm{H})$, 
1.33-1.26 (m, 2H), $0.90(\mathrm{t}, J 7.2 \mathrm{~Hz}, 3 \mathrm{H}) ;{ }^{13} \mathrm{C} \mathrm{NMR}\left(\mathrm{CDCl}_{3}, 100 \mathrm{MHz}\right) 167.9,165.1,135.6$, 135.5, 129.0, 128.9, 128.6, 128.1, 128.0, 126.4, 63.4, 55.2, 47.3, 45.3, 29.2, 20.0, 18.7, 13.7; HRMS calcd for $\mathrm{C}_{22} \mathrm{H}_{26} \mathrm{~N}_{2} \mathrm{O}_{2}\left(\mathrm{M}^{+}\right)$: 350.1994. Found 350.1991.

(3S,6S)-1-Benzyl-4-isobutyl-6-methyl-3-phenylpiperazine-2,5-dione (5) A colorless oil was obtained in $66 \%$ yield. ${ }^{1} \mathrm{H}$ NMR $\left(\mathrm{CDCl}_{3}, 400 \mathrm{MHz}\right) 7.46-7.15(\mathrm{~m}, 14 \mathrm{H}), 5.25(\mathrm{~s}, 1 \mathrm{H}), 5.20(\mathrm{~d}, J$ $14.8 \mathrm{~Hz}, 1 \mathrm{H}), 4.11-3.91(\mathrm{~m}, 3 \mathrm{H}), 2.43-2.31(\mathrm{~m}, 1 \mathrm{H}), 2.10-1.99(\mathrm{~m}, 1 \mathrm{H}), 1.34(\mathrm{~d}, 1 \mathrm{H}), 0.95-0.84$ $(\mathrm{m}, 6 \mathrm{H}) ;{ }^{13} \mathrm{C} \mathrm{NMR}\left(\mathrm{CDCl}_{3}, 100 \mathrm{MHz}\right) 168.6,165.1,135.7,135.5,129.0,128.9,128.5,127.9$, 127.8, 126.2, 64.0, 55.1, 52.5, 47.2, 26.5, 20.1, 19.8, 18.4; HRMS calcd for $\mathrm{C}_{22} \mathrm{H}_{26} \mathrm{~N}_{2} \mathrm{O}_{2}\left(\mathrm{M}^{+}\right)$: 350.1994. Found 350.1992.

(3S,6S)-1-Benzyl-6-methyl-4-(2-phenylethyl)-3-phenylpiperazine-2,5-dione (6) A colorless oil was obtained in $75 \%$ yield. ${ }^{1} \mathrm{H}$ NMR $\left(\mathrm{CDCl}_{3}, 400 \mathrm{MHz}\right) 7.38-7.11(\mathrm{~m}, 15 \mathrm{H}), 5.22(\mathrm{~d}, J 15.2$ $\mathrm{Hz}, 1 \mathrm{H}), 4.88(\mathrm{~s}, 1 \mathrm{H}), 4.35-4.30(\mathrm{~m}, 1 \mathrm{H}), 3.95(\mathrm{q}, J 6.8 \mathrm{~Hz}, 1 \mathrm{H}), 3.83(\mathrm{~d}, J 15.2 \mathrm{~Hz}, 1 \mathrm{H}), 2.95-$ $2.77(\mathrm{~m}, 3 \mathrm{H}), 1.37(\mathrm{~d}, J 6.8 \mathrm{~Hz}, 3 \mathrm{H}) ;{ }^{13} \mathrm{C} \mathrm{NMR}\left(\mathrm{CDCl}_{3}, 100 \mathrm{MHz}\right) 167.9,164.7,138.1,135.6$, 129.2, 129.0, 128.9, 128.8, 128.7, 128.6, 128.1, 127.2, 126.8, 126.4, 64.3, 54.8, 47.5, 47.1, 33.5, 18.6; HRMS calcd for $\mathrm{C}_{26} \mathrm{H}_{26} \mathrm{~N}_{2} \mathrm{O}_{2}\left(\mathrm{M}^{+}\right)$: 398.1994. Found 398.1995.

(3S,6S)-1-Benzyl-4,6-dimethyl-3-phenylpiperazine-2,5-dione (7) A pale yellow oil was obtained in 51\% yield. ${ }^{1} \mathrm{H}$ NMR $\left(\mathrm{CDCl}_{3}, 400 \mathrm{MHz}\right) 7.42-7.22(\mathrm{~m}, 10 \mathrm{H}), 5.19(\mathrm{~d}, J 14.8 \mathrm{~Hz}, 1 \mathrm{H})$, 5.13 (s, 1H), 4.00 (q, J 7.2 Hz, 1H), 3.90 (d, J $14.8 \mathrm{~Hz}, 1 \mathrm{H}), 2.96(\mathrm{~s}, 3 \mathrm{H}), 1.43$ (d, J 7.2 Hz, 3H); ${ }^{13} \mathrm{C} \mathrm{NMR}\left(\mathrm{CDCl}_{3}, 100 \mathrm{MHz}\right) 167.9,164.6,135.6,135.2,129.1,128.9,128.6,128.3,128.0$, 126.3, 65.9, 54.9, 47.2, 33.1, 19.0; HRMS calcd for $\mathrm{C}_{19} \mathrm{H}_{21} \mathrm{~N}_{2} \mathrm{O}_{2}\left(\mathrm{M}^{+}+1\right)$ : 309.1603. Found 309.1603 .

(3S,6S)-1-Benzyl-4-methoxycarbonylmethyl-6-methyl-3-phenyl piperazine-2,5-dione (8) A colorless oil was obtained in $76 \%$ yield. ${ }^{1} \mathrm{H}$ NMR $\left(\mathrm{CDCl}_{3}, 400 \mathrm{MHz}\right) 7.46-7.22(\mathrm{~m}, 10 \mathrm{H}), 5.30$ $(\mathrm{s}, 1 \mathrm{H}), 5.18(\mathrm{~d}, J 15.2 \mathrm{~Hz}, 1 \mathrm{H}), 4.78(\mathrm{~d}, J 17.6 \mathrm{~Hz}, 1 \mathrm{H}), 4.07-4.00(\mathrm{~m}, 2 \mathrm{H}), 3.70(\mathrm{~s}, 3 \mathrm{H}), 3.42(\mathrm{~d}$, $J 17.6 \mathrm{~Hz}, 1 \mathrm{H}), 1.44$ (d, $J 7.2 \mathrm{~Hz}, 3 \mathrm{H}) ;{ }^{13} \mathrm{C} \mathrm{NMR}\left(\mathrm{CDCl}_{3}, 100 \mathrm{MHz}\right) 168.3,168.0,164.3,135.4$, $134.8,129.2$, 129.0, 128.9, 128.1, 128.0, 126.6, 64.7, 54.8, 52.5, 47.4, 46.1, 19.3; HRMS calcd for $\mathrm{C}_{21} \mathrm{H}_{22} \mathrm{~N}_{2} \mathrm{O}_{4}\left(\mathrm{M}^{+}\right)$: 366.1580. Found 366.1579.

(3S,6S)-4-( $N$-Boc-2-aminoethyl)-1-benzyl-6-methyl-3-phenyl piperazine-2,5-dione (9) A colorless oil was obtained in $60 \%$ yield. ${ }^{1} \mathrm{H}$ NMR $\left(\mathrm{CDCl}_{3}, 400 \mathrm{MHz}\right) 7.42-7.22(\mathrm{~m}, 10 \mathrm{H}), 5.29$ (s, 1H), 5.11 (d, J $15.2 \mathrm{~Hz}, 1 \mathrm{H}), 4.97$ (br, 1H), 4.08-3.98 (m, 3H), 3.33-3.30 (m, 1H), 2.94-2.88 $(\mathrm{m}, 1 \mathrm{H}), 1.43-1.36(\mathrm{~m}, 12 \mathrm{H}) ;{ }^{13} \mathrm{C} \mathrm{NMR}\left(\mathrm{CDCl}_{3}, 100 \mathrm{MHz}\right) 168.6,164.6,156.0,135.6,135.4$, 129.1, 128.9, 128.2, 128.0, 126.4, 79.6, 64.6, 55.1, 47.4, 45.7, 38.7, 28.4, 18.9; HRMS calcd for $\mathrm{C}_{25} \mathrm{H}_{32} \mathrm{~N}_{3} \mathrm{O}_{4}\left(\mathrm{M}^{+}+1\right): 438.2393$. Found 438.2388.

(3S,6S)-1-Benzyl-4-(3-indoleethyl)-6-methyl-3-phenylpiperazine-2,5-dione (10) A pale yellow oil was obtained in $83 \%$ yield. ${ }^{1} \mathrm{H}$ NMR $\left(\mathrm{CDCl}_{3}, 400 \mathrm{MHz}\right) 8.40(\mathrm{br}, 1 \mathrm{H}), 7.54-6.84(\mathrm{~m}$, $15 \mathrm{H}), 5.09$ (d, $J 14.8 \mathrm{~Hz}, 1 \mathrm{H}), 4.99$ (s, 1H), 4.37-4.31 (m, 1H), 3.99 (q, $J 7.2 \mathrm{~Hz}, 1 \mathrm{H}), 3.91$ (d, $J$ $14.8 \mathrm{~Hz}, 1 \mathrm{H}), 3.13-2.88(\mathrm{~m}, 3 \mathrm{H}), 1.38(\mathrm{~d}, J 7.2 \mathrm{~Hz}, 3 \mathrm{H}) ;{ }^{13} \mathrm{C} \mathrm{NMR}\left(\mathrm{CDCl}_{3}, 100 \mathrm{MHz}\right) 167.9$, $164.9,136.3,135.7,135.6,129.0,128.9,128.6,128.3,128.1,127.1,126.5,122.2,122.1,119.5$, $118.5,112.0,111.5,64.4,55.2,47.4,46.8,23.2$, 18.8; HRMS calcd for $\mathrm{C}_{28} \mathrm{H}_{27} \mathrm{~N}_{3} \mathrm{O}_{2}\left(\mathrm{M}^{+}\right)$: 437.2103. Found 437.2103. 
(3S,6S)-1-Benzyl-4-(4-methoxybenzyl)-6-methyl-3-phenylpiperazine-2,5-dione (11) A pale yellow oil was obtained in 77\% yield. ${ }^{1} \mathrm{H}$ NMR $\left(\mathrm{CDCl}_{3}, 400 \mathrm{MHz}\right) 7.45-6.82(\mathrm{~m}, 14 \mathrm{H}), 5.52(\mathrm{~d}$, $J 14.8 \mathrm{~Hz}, 1 \mathrm{H}), 5.10$ (d, $J 15.2 \mathrm{~Hz}, 1 \mathrm{H}), 5.08(\mathrm{~s}, 1 \mathrm{H}), 4.05$ (q, $J 6.8 \mathrm{~Hz}, 1 \mathrm{H}), 3.96$ (d, $J 15.2 \mathrm{~Hz}$, $1 \mathrm{H}), 3.77(\mathrm{~s}, 3 \mathrm{H}), 3.53(\mathrm{~d}, J 14.8 \mathrm{~Hz}, 1 \mathrm{H}), 1.44(\mathrm{~d}, J 6.8 \mathrm{~Hz}, 3 \mathrm{H}) ;{ }^{13} \mathrm{C} \mathrm{NMR}\left(\mathrm{CDCl}_{3}, 100 \mathrm{MHz}\right)$ $167.9,164.9,159.5,135.7,135.2,129.9,129.1,128.9,128.6,128.0,127.9,127.2$, 126.6, 114.3, 62.0, 55.3, 55.2, 47.3, 47.0, 19.1; HRMS calcd for $\mathrm{C}_{26} \mathrm{H}_{26} \mathrm{~N}_{2} \mathrm{O}_{3}\left(\mathrm{M}^{+}\right)$: 414.1943. Found 414.1942. (3S,6S)-1,6-Dibenzyl-4-(2-phenylethyl)-3-phenylpiperazine-2,5-dione (20) A colorless oil was obtained in 56\% yield. ${ }^{1} \mathrm{H}$ NMR $\left(\mathrm{CDCl}_{3}, 400 \mathrm{MHz}\right) 7.26-6.92(\mathrm{~m}, 20 \mathrm{H}), 5.20(\mathrm{~d}, J 14.8 \mathrm{~Hz}, 1 \mathrm{H})$, $4.71(\mathrm{~s}, 1 \mathrm{H}), 4.17-4.05(\mathrm{~m}, 2 \mathrm{H}), 3.15-3.09(\mathrm{~m}, 2 \mathrm{H}), 2.88-2.61(\mathrm{~m}, 4 \mathrm{H}) ;{ }^{13} \mathrm{C} \mathrm{NMR}\left(\mathrm{CDCl}_{3}, 100\right.$ MHz) 166.4, 165.1, 138.2, 136.5, 135.5, 135.4, 129.7, 129.0, 128.9, 128.8, 128.7, 128.5, 128.4, $128.1,127.4,126.9,126.6,64.5,60.5,47.7,47.6,39.2$, 33.4; HRMS: calcd. for $\mathrm{C}_{32} \mathrm{H}_{31} \mathrm{~N}_{2} \mathrm{O}_{2}$ $\left(\mathrm{M}^{+}+1\right)$ 475.2386; Found 475.2386.

(3S,6S)-1-Benzyl-6-isobutyl-4-(2-phenylethyl)-3-phenylpiperazine-2,5-dione (21) A colorless oil was obtained in $70 \%$ yield. ${ }^{1} \mathrm{H}$ NMR $\left(\mathrm{CDCl}_{3}, 400 \mathrm{MHz}\right) 7.38-7.12(\mathrm{~m}, 15 \mathrm{H}), 5.26(\mathrm{~d}, J 14.8$ $\mathrm{Hz}, 1 \mathrm{H}), 4.90$ (s, 1H), 4.39-4.34 (m, 1H), 3.89-3.85 (m, 1H), 3.74 (d, J 14.8 Hz, 1H), 2.95-2.80 (m, 3H), 1.91-1.86 (m, 1H), 1.48-1.27 (m, 2H), $0.89(\mathrm{~d}, J 6.4 \mathrm{~Hz}, 3 \mathrm{H}), 0.82(\mathrm{~d}, J 6.4 \mathrm{~Hz}, 3 \mathrm{H}) ;{ }^{13} \mathrm{C}$ NMR $\left(\mathrm{CDCl}_{3}, 100 \mathrm{MHz}\right)$ 167.3, 165.3, 138.1, 135.8, 135.3, 129.0, 128.9, 128.8, 128.7, 128.5, 128.2, 128.1, 126.8, 126.1, 64.2, 57.3, 47.8, 47.6, 42.5, 33.5, 25.0, 23.2, 21.5; HRMS: calcd. for $\mathrm{C}_{29} \mathrm{H}_{33} \mathrm{~N}_{2} \mathrm{O}_{2}\left(\mathrm{M}^{+}+1\right)$ 441.2542; Found 441.2542.

(3S,6S)-1-Benzyl-6-methoxycarbonylmethyl-4-(2-phenylethyl)-3-phenylpiperazine-2,5-

dione (22) A pale yellow oil was obtained in 71\% yield. ${ }^{1} \mathrm{H}$ NMR $\left(\mathrm{CDCl}_{3}, 400 \mathrm{MHz}\right) 7.39-7.09$ (m, 15H), 5.09 (d, J $14.8 \mathrm{~Hz}, 1 \mathrm{H}), 4.86(\mathrm{~s}, 1 \mathrm{H}), 4.50$ (t, J 6.0 Hz, 1H), 4.37-4.25 (m, 1H), 3.98 $(\mathrm{d}, J 14.8 \mathrm{~Hz}, 1 \mathrm{H}), 3.65(\mathrm{~s}, 3 \mathrm{H}), 2.97-2.72(\mathrm{~m}, 4 \mathrm{H}), 2.51-2.46(\mathrm{~m}, 1 \mathrm{H}) ;{ }^{13} \mathrm{C} \mathrm{NMR}\left(\mathrm{CDCl}_{3}, 100\right.$ MHz) 170.4, 166.3, 164.9, 138.0, 135.7, 135.2, 129.2, 128.9, 128.8, 128.7, 128.2, 128.0, 126.9, 126.3, 64.3, 56.2, 52.3, 47.7, 38.9, 33.5; HRMS calcd for $\mathrm{C}_{28} \mathrm{H}_{29} \mathrm{~N}_{2} \mathrm{O}_{4}\left(\mathrm{M}^{+}+1\right)$ : 457.2127. Found 457.2123.

(3S,6S)-1-Benzyl-6-(2-methoxycarbonylethyl)-4-(2-phenylethyl)-3-phenylpiperazine-2,5-

dione (23) A colorless oil was obtained in $81 \%$ yield. ${ }^{1} \mathrm{H}$ NMR $\left(\mathrm{CDCl}_{3}, 400 \mathrm{MHz}\right) 7.40-7.23$ (m, $15 \mathrm{H}), 5.23$ (d, J $14.8 \mathrm{~Hz}, 1 \mathrm{H}), 4.85(\mathrm{~s}, 1 \mathrm{H}), 4.52-4.27$ (m, 2H), 3.98-3.77 (m, 2H), 3.67 (s, 3H), 2.95-2.38 (m, 5H), 2.09-2.06 (m, 1H), 1.73-1.67 (m, 1H); $\left.{ }^{13} \mathrm{C} \mathrm{NMR} \mathrm{(CDCl} 3,100 \mathrm{MHz}\right) 172.9$, $166.5,164.8,138.0,135.8,135.2,130.0,129.0,128.9,128.7,128.6,128.5,128.0,126.8,126.1$, 64.2, 57.8, 51.8, 47.7, 47.3, 33.4, 29.8, 27.4; HRMS: calcd. for $\mathrm{C}_{29} \mathrm{H}_{31} \mathrm{~N}_{2} \mathrm{O}_{4}\left(\mathrm{M}^{+}+1\right)$ 471.2284; Found 471.2284.

(3S,6S)-1-Benzyl-3-(4-chlorophenyl)-6-isobutyl-4-(2-phenylethyl) piperazine-2,5-dione (24) A pale yellow oil was obtained in $48 \%$ yield. ${ }^{1} \mathrm{H} \mathrm{NMR}\left(\mathrm{CDCl}_{3}, 400 \mathrm{MHz}\right) 7.39-7.11(\mathrm{~m}, 14 \mathrm{H})$, $5.24(\mathrm{~d}, J 14.8 \mathrm{~Hz}, 1 \mathrm{H}), 4.82(\mathrm{~s}, 1 \mathrm{H}), 4.39-4.32(\mathrm{~m}, 1 \mathrm{H}), 3.89-3.85(\mathrm{~m}, 1 \mathrm{H}), 3.76(\mathrm{~d}, J 14.8 \mathrm{~Hz}$, $1 \mathrm{H}), 2.98-2.74(\mathrm{~m}, 3 \mathrm{H}), 1.91-1.84(\mathrm{~m}, 1 \mathrm{H}), 1.44-1.25(\mathrm{~m}, 2 \mathrm{H}), 0.88$ (d, $J 6.4 \mathrm{~Hz}, 3 \mathrm{H}), 0.80$ (d, $J$ $6.4 \mathrm{~Hz}, 3 \mathrm{H}) ;{ }^{13} \mathrm{C} \mathrm{NMR}\left(\mathrm{CDCl}_{3}, 100 \mathrm{MHz}\right) 167.1,164.9,138.0,135.6,134.6,133.8,129.1,129.0$, $128.8,128.7,128.2,128.1,127.5,126.9,63.7,57.3,47.7,47.6,42.7,33.5,25.0,23.1,21.5$; HRMS: calcd. for $\mathrm{C}_{29} \mathrm{H}_{32} \mathrm{ClN}_{2} \mathrm{O}_{2}\left(\mathrm{M}^{+}+1\right)$ 475.2152; Found 475.2152. 
(3S,6S)-1-Benzyl-3-(4-bromophenyl)-6-isobutyl-4-(2-phenylethyl) piperazine-2,5-dione (25) A colorless oil was obtained in $51 \%$ yield. ${ }^{1} \mathrm{H}$ NMR $\left(\mathrm{CDCl}_{3}, 400 \mathrm{MHz}\right) 7.51-7.08(\mathrm{~m}, 14 \mathrm{H}), 5.23$ $(\mathrm{d}, J 14.8 \mathrm{~Hz}, 1 \mathrm{H}), 4.80(\mathrm{~s}, 1 \mathrm{H}), 4.37-4.32(\mathrm{~m}, 1 \mathrm{H}), 3.89-3.85(\mathrm{~m}, 1 \mathrm{H}), 3.76(\mathrm{~d}, J 14.8 \mathrm{~Hz}, 1 \mathrm{H})$, 2.96-2.76 (m, 3H), 1.89-1.86 (m, 1H), 1.41-1.32 (m, 2H), $0.90(\mathrm{~d}, J 6.4 \mathrm{~Hz}, 3 \mathrm{H}), 0.82(\mathrm{~d}, J 6.4$ $\mathrm{Hz}, 3 \mathrm{H}) ;{ }^{13} \mathrm{C} \mathrm{NMR}\left(\mathrm{CDCl}_{3}, 100 \mathrm{MHz}\right) 167.1,164.8,138.0,135.6,134.4,132.0,129.0,128.8$, $128.7,128.2,128.1,127.9,126.9,122.7,63.8,57.3,47.7,47.6,42.7,33.5,25.0,23.2,21.5$; HRMS: calcd. for $\mathrm{C}_{29} \mathrm{H}_{32} \mathrm{BrN}_{2} \mathrm{O}_{2}\left(\mathrm{M}^{+}+1\right)$ 519.1647; Found 519.1647.

(3S,6S)-1-Benzyl-6-isobutyl-3-methyl-4-(2-phenylethyl)piperazine-2,5-dione (26) A colorless oil was obtained in $89 \%$ yield. ${ }^{1} \mathrm{H}$ NMR $\left(\mathrm{CDCl}_{3}, 400 \mathrm{MHz}\right) 7.37-7.15(\mathrm{~m}, 10 \mathrm{H}), 5.33(\mathrm{~d}, J 14.8$ $\mathrm{Hz}, 1 \mathrm{H}), 4.02-3.96(\mathrm{~m}, 1 \mathrm{H}), 3.83-3.75(\mathrm{~m}, 3 \mathrm{H}), 3.11-3.04(\mathrm{~m}, 1 \mathrm{H}), 2.94-2.86(\mathrm{~m}, 2 \mathrm{H}), 1.91-1.89$ $(\mathrm{m}, 1 \mathrm{H}), 1.71-1.55(\mathrm{~m}, 2 \mathrm{H}), 1.48(\mathrm{~d}, J 7.2 \mathrm{~Hz}, 3 \mathrm{H}), 0.95-0.92(\mathrm{~m}, 6 \mathrm{H}) ;{ }^{13} \mathrm{C} \mathrm{NMR}\left(\mathrm{CDCl}_{3}, 100\right.$ MHz) 167.4, 166.3, 138.3, 135.7, 128.9, 128.8, 128.7, 128.2, 128.0, 126.7, 56.9, 47.1, 46.7, 43.3, 33.4, 25.1, 23.4, 21.7, 19.4; HRMS: calcd. for $\mathrm{C}_{24} \mathrm{H}_{31} \mathrm{~N}_{2} \mathrm{O}_{2}\left(\mathrm{M}^{+}+1\right)$ 379.2386; Found 379.2386.

(3S,6S)-1-Benzyl-3-ethyl-6-isobutyl-4-(2-phenylethyl)piperazine-2,5-dione (27) A pale yellow oil was obtained in $75 \%$ yield. ${ }^{1} \mathrm{H} \mathrm{NMR}\left(\mathrm{CDCl}_{3}, 400 \mathrm{MHz}\right) 7.35-7.14(\mathrm{~m}, 10 \mathrm{H}), 5.30$ (d, $J 14.8 \mathrm{~Hz}, 1 \mathrm{H}), 4.11-4.05$ (m, 1H), 3.80 (d, $J 14.8 \mathrm{~Hz}, 1 \mathrm{H}), 3.80-3.77$ (m, 1H), 3.65-3.61 (m, $1 \mathrm{H}), 3.06-2.81(\mathrm{~m}, 3 \mathrm{H}), 1.98-1.69(\mathrm{~m}, 4 \mathrm{H}), 1.57-1.50(\mathrm{~m}, 1 \mathrm{H}), 1.06(\mathrm{t}, J 7.2 \mathrm{~Hz}, 3 \mathrm{H}), 0.94-0.91$ $(\mathrm{m}, 6 \mathrm{H}) ;{ }^{13} \mathrm{C} \mathrm{NMR}\left(\mathrm{CDCl}_{3}, 100 \mathrm{MHz}\right) 166.7,166.3,138.3,135.9,128.9,128.7,128.6,128.2$, 128.0, 126.7, 62.4, 57.1, 47.3, 47.0, 43.8, 33.4, 27.4, 25.5, 23.3, 21.7, 11.1; HRMS: calcd. for $\mathrm{C}_{25} \mathrm{H}_{33} \mathrm{~N}_{2} \mathrm{O}_{2}\left(\mathrm{M}^{+}+1\right)$ 393.2542; Found 393.2543.

(3S,6S)-4-Benzyl-6-isobutyl-1-(4-methoxybenzyl)-3-phenyl piperazine-2,5-dione (35) A colorless oil was obtained in $70 \%$ yield. ${ }^{1} \mathrm{H}$ NMR $\left(\mathrm{CDCl}_{3}, 400 \mathrm{MHz}\right) 7.47-6.83(\mathrm{~m}, 14 \mathrm{H}), 5.61$ (d, $J 14.8 \mathrm{~Hz}, 1 \mathrm{H}), 5.20(\mathrm{~d}, J 14.8 \mathrm{~Hz}, 1 \mathrm{H}), 5.12(\mathrm{~s}, 1 \mathrm{H}), 3.98-3.95(\mathrm{~m}, 1 \mathrm{H}), 3.81(\mathrm{~s}, 3 \mathrm{H}), 3.76(\mathrm{~d}$, $J 14.8 \mathrm{~Hz}, 1 \mathrm{H}), 3.61(\mathrm{~d}, J 14.8 \mathrm{~Hz}, 1 \mathrm{H}), 1.96-1.90(\mathrm{~m}, 1 \mathrm{H}), 1.62-1.51(\mathrm{~m}, 1 \mathrm{H}), 1.41-1.34(\mathrm{~m}$, $1 \mathrm{H}), 0.92(\mathrm{~d}, J 6.4 \mathrm{~Hz}, 3 \mathrm{H}), 0.85(\mathrm{~d}, J 6.8 \mathrm{~Hz}, 3 \mathrm{H}) ;{ }^{13} \mathrm{C} \mathrm{NMR}\left(\mathrm{CDCl}_{3}, 100 \mathrm{MHz}\right) 167.5,165.5$, $159.4,135.5,134.9,129.4,129.0,128.9,128.5,128.2,128.1,127.7,126.3,114.3,62.4,57.3$, 55.3, 47.8, 47.2, 42.8, 25.0, 23.1, 21.6; HRMS calcd for $\mathrm{C}_{29} \mathrm{H}_{32} \mathrm{~N}_{2} \mathrm{O}_{3}\left(\mathrm{M}^{+}\right)$: 456.2413. Found 456.2415 .

(3S,6S)-4-Benzyl-1-(4-methoxybenzyl)-6-methyl-3-phenylpiperazine-2,5-dione (36) A colorless oil was obtained in 73\% yield. ${ }^{1} \mathrm{H}$ NMR $\left(\mathrm{CDCl}_{3}, 400 \mathrm{MHz}\right) 7.45-7.25(\mathrm{~m}, 8 \mathrm{H}), 7.15-$ $7.11(\mathrm{~m}, 4 \mathrm{H}), 6.84-6.82(\mathrm{~m}, 2 \mathrm{H}), 5.58$ (d, $J 14.8 \mathrm{~Hz}, 1 \mathrm{H}), 5.08$ (d, $J 14.8 \mathrm{~Hz}, 1 \mathrm{H}), 5.07(\mathrm{~s}, 1 \mathrm{H})$, 4.06 (q, J 7.2 Hz, 1H), 3.89 (d, J $14.8 \mathrm{~Hz}, 1 \mathrm{H}), 3.78$ (s, 3H), 3.59 (d, $J 14.8 \mathrm{~Hz}, 1 \mathrm{H}), 1.45$ (d, $J$ $7.2 \mathrm{~Hz}, 3 \mathrm{H}) ;{ }^{13} \mathrm{C} \mathrm{NMR}\left(\mathrm{CDCl}_{3}, 100 \mathrm{MHz}\right) 168.1,164.7,159.4,135.2,135.1,129.5,129.1,128.9$, 128.7, 128.4, 128.1, 127.6, 126.6, 114.3, 62.4, 55.3, 54.9, 47.6, 46.8, 19.1; HRMS: calcd. for $\mathrm{C}_{26} \mathrm{H}_{27} \mathrm{~N}_{2} \mathrm{O}_{3}\left(\mathrm{M}^{+}+1\right)$ 415.2022; Found 415.2022.

(3S,6S)-6-Methyl-1-(4-methoxybenzyl)-3-phenyl-4-(n-propyl) piperazine-2,5-dione (37) A colorless oil was obtained in $80 \%$ yield. ${ }^{1} \mathrm{H} \mathrm{NMR}\left(\mathrm{CDCl}_{3}, 400 \mathrm{MHz}\right) 7.45-6.82(\mathrm{~m}, 9 \mathrm{H}), 5.20$ (s, $1 \mathrm{H}), 5.10$ (d, $J 14.8 \mathrm{~Hz}, 1 \mathrm{H}), 4.07-3.92(\mathrm{~m}, 2 \mathrm{H}), 3.87$ (d, J $14.8 \mathrm{~Hz}, 1 \mathrm{H}), 3.75$ (s, 3H), 2.65-2.58 $(\mathrm{m}, 1 \mathrm{H}), 1.64-1.53(\mathrm{~m}, 2 \mathrm{H}), 1.36(\mathrm{~d}, J 6.8 \mathrm{~Hz}, 3 \mathrm{H}), 0.87(\mathrm{t}, J 7.2 \mathrm{~Hz}, 3 \mathrm{H}) ;{ }^{13} \mathrm{C} \mathrm{NMR}\left(\mathrm{CDCl}_{3}, 100\right.$ 
MHz) 167.9, 164.8, 159.3, 135.6, 129.6, 128.9, 128.5, 127.6, 126.3, 114.2, 63.4, 55.2, 54.8, 46.9, 46.6, 20.4, 18.6, 11.2; HRMS: calcd. for $\mathrm{C}_{22} \mathrm{H}_{27} \mathrm{~N}_{2} \mathrm{O}_{3}\left(\mathrm{M}^{+}+1\right)$ 367.2022; found 367.2022.

(3S,6S)-1,4-Di(4-methoxybenzyl)-6-methyl-3-phenylpiperazine-2,5-dione (38) A colorless oil was obtained in $68 \%$ yield. ${ }^{1} \mathrm{H}$ NMR $\left(\mathrm{CDCl}_{3}, 400 \mathrm{MHz}\right) 7.45-7.25(\mathrm{~m}, 5 \mathrm{H}), 7.11-7.05(\mathrm{~m}, 4 \mathrm{H})$, 6.84-6.80 (m, 4H), 5.50 (d, $J 14.4 \mathrm{~Hz}, 1 \mathrm{H}), 5.07$ (s, 1H), 5.03 (d, J $14.8 \mathrm{~Hz}, 1 \mathrm{H}), 4.05$ (q, $J 7.2$ Hz, 1H), 3.89 (d, $J 14.8 \mathrm{~Hz}, 1 \mathrm{H}), 3.74-3.71(\mathrm{~m}, 6 \mathrm{H}), 3.53$ (d, $J 14.8 \mathrm{~Hz}, 1 \mathrm{H}), 1.44(\mathrm{~d}, J 7.2 \mathrm{~Hz}$, $3 \mathrm{H}) ;{ }^{13} \mathrm{C} \mathrm{NMR}\left(\mathrm{CDCl}_{3}, 100 \mathrm{MHz}\right) 167.9,164.7,159.5,135.2,129.9,129.5,129.1,129.0,128.6$, 127.7, 127.3, 127.2, 126.6, 114.3, 114.2, 62.1, 55.3, 55.2, 54.9, 47.0, 46.7, 19.1; HRMS calcd for $\mathrm{C}_{27} \mathrm{H}_{28} \mathrm{~N}_{2} \mathrm{O}_{4}\left(\mathrm{M}^{+}\right):$444.2049. Found 444.2047.

(3S,6S)-1,4-Di(4-methoxybenzyl)-3,6-dimethylpiperazine-2,5-dione (39) A colorless oil was obtained in $60 \%$ yield. ${ }^{1} \mathrm{H}$ NMR $\left(\mathrm{CDCl}_{3}, 400 \mathrm{MHz}\right) 7.15(\mathrm{~d}, J 8.4 \mathrm{~Hz}, 4 \mathrm{H}), 6.85(\mathrm{~d}, J 8.4 \mathrm{~Hz}$, $4 \mathrm{H}), 5.08$ (d, $J 14.8 \mathrm{~Hz}, 2 \mathrm{H}), 4.02-3.93(\mathrm{~m}, 4 \mathrm{H}), 3.79$ (s, 6H), 1.49 (d, $J 7.2 \mathrm{~Hz}, 6 \mathrm{H}) ;{ }^{13} \mathrm{C} \mathrm{NMR}$ $\left(\mathrm{CDCl}_{3}, 100 \mathrm{MHz}\right) 167.1,159.4,129.6,127.8,114.6,55.3,54.9,46.6,19.1$. The spectral data of 39 were identical to those of the authentic material reported previously. ${ }^{26}$

(3S,6S)-1-Cinnamyl-6-isobutyl-4-(2-phenylethyl)-3-phenylpiperazine-2,5-dione (40) A yellow oil was obtained in 56\% yield. ${ }^{1} \mathrm{H}$ NMR $\left(\mathrm{CDCl}_{3}, 400 \mathrm{MHz}\right) 7.42-7.16(\mathrm{~m}, 15 \mathrm{H}), 6.52(\mathrm{~d}$, $J 16.0 \mathrm{~Hz}, 1 \mathrm{H}), 6.11-6.05(\mathrm{~m}, 1 \mathrm{H}), 4.96(\mathrm{~s}, 1 \mathrm{H}), 4.72-4.67(\mathrm{~m}, 1 \mathrm{H}), 4.37-4.32(\mathrm{~m}, 1 \mathrm{H}), 4.04-3.94$ (m, 1H), 3.54-3.48 (m, 1H), 2.96-2.82 (m, 3H), 1.93-1.80 (m, 1H), 1.45-1.34 (m, 2H), $0.92(\mathrm{~d}, J$ $6.4 \mathrm{~Hz}, 3 \mathrm{H}), 0.80$ (d, $J 6.8 \mathrm{~Hz}, 3 \mathrm{H}) ;{ }^{13} \mathrm{C} \mathrm{NMR}\left(\mathrm{CDCl}_{3}, 100 \mathrm{MHz}\right) 167.3,165.0,138.1,136.1$, $135.3,134.5,128.9,128.7,128.6,128.5,128.4,128.1,126.7,126.5,126.1,122.9,64.2,57.6$, 47.8, 46.8, 42.8, 33.5, 25.0, 23.1, 21.6; HRMS calcd for $\mathrm{C}_{31} \mathrm{H}_{35} \mathrm{~N}_{2} \mathrm{O}_{2}\left(\mathrm{M}^{+}+1\right)$ : 467.2698. Found 467.2691.

(3S,6S)-1-Cinnamyl-4-(4-methoxybenzyl)-6-methyl-3-phenyl piperazine-2,5-dione (41) A yellow oil was obtained in 77\% yield. ${ }^{1} \mathrm{H}$ NMR $\left(\mathrm{CDCl}_{3}, 400 \mathrm{MHz}\right) 7.37-6.82(\mathrm{~m}, 14 \mathrm{H}), 6.47(\mathrm{~d}$, $J 16.0 \mathrm{~Hz}, 1 \mathrm{H}), 6.11-6.04(\mathrm{~m}, 1 \mathrm{H}), 5.53(\mathrm{~d}, J 14.8 \mathrm{~Hz}, 1 \mathrm{H}), 5.04(\mathrm{~s}, 1 \mathrm{H}), 4.58-4.52(\mathrm{~m}, 1 \mathrm{H}), 4.20$ $(\mathrm{q}, J 7.2 \mathrm{~Hz}, 1 \mathrm{H}), 3.80(\mathrm{~s}, 3 \mathrm{H}), 3.70-3.64(\mathrm{~m}, 1 \mathrm{H}), 3.51(\mathrm{~d}, J 14.8 \mathrm{~Hz}, 1 \mathrm{H}), 1.53$ (d, J 7.2 Hz, $3 \mathrm{H}) ;{ }^{13} \mathrm{C} \mathrm{NMR}\left(\mathrm{CDCl}_{3}, 100 \mathrm{MHz}\right)$ 167.8, 164.4, 159.5, 136.0, 135.1, 134.2, 130.1, 129.1, 128.6, $128.1,126.7,126.5,122.7,114.3,114.2,62.0,55.5,55.3,47.0,46.4,19.4$; HRMS: calcd. for $\mathrm{C}_{28} \mathrm{H}_{29} \mathrm{~N}_{2} \mathrm{O}_{3}\left(\mathrm{M}^{+}+1\right)$ 441.2178; Found 441.2178.

(3R,8aS)-2-(2-Phenylethyl)-3-phenylhexahydropyrrolo[1,2-a]pyrazine -1,4-dione (42) A colorless oil was obtained in $66 \%$ yield. ${ }^{1} \mathrm{H}$ NMR $\left(\mathrm{CDCl}_{3}, 400 \mathrm{MHz}\right) 7.39-7.15(\mathrm{~m}, 10 \mathrm{H}), 5.00$ $(\mathrm{s}, 1 \mathrm{H}), 4.33-4.26(\mathrm{~m}, 1 \mathrm{H}), 4.01-3.97(\mathrm{~m}, 1 \mathrm{H}), 3.63-3.56(\mathrm{~m}, 1 \mathrm{H}), 3.42-3.37(\mathrm{~m}, 1 \mathrm{H}), 3.03-2.84$ (m, 3H), 2.38-2.32 (m, 1H), 2.05-1.92 (m, 2H), 1.80-1.78 (m, 1H); $\left.{ }^{13} \mathrm{C} \mathrm{NMR} \mathrm{(CDCl} 3,100 \mathrm{MHz}\right)$ $168.1,164.1,138.0,134.4,129.2,128.8,128.6,126.7,125.7,66.2,58.2,47.2$, 45.7, 34.0, 29.2, 22.5; HRMS calcd for $\mathrm{C}_{21} \mathrm{H}_{23} \mathrm{~N}_{2} \mathrm{O}_{2}\left(\mathrm{M}^{+}+1\right)$ : 335.1760. Found 335.1762.

(3R,8aS)-2-(4-Methoxybenzyl)-3-phenylhexahydropyrrolo[1,2-a] pyrazine-1,4-dione (43) A colorless oil was obtained in $87 \%$ yield. ${ }^{1} \mathrm{H}$ NMR $\left(\mathrm{CDCl}_{3}, 400 \mathrm{MHz}\right) 7.42-6.81(\mathrm{~m}, 9 \mathrm{H}), 5.45(\mathrm{~d}$, $J 14.4 \mathrm{~Hz}, 1 \mathrm{H}), 4.99(\mathrm{~s}, 1 \mathrm{H}), 4.17-4.13(\mathrm{~m}, 1 \mathrm{H}), 3.81(\mathrm{~s}, 3 \mathrm{H}), 3.66-3.59$ (m, 2H), 3.43-3.38 (m, $1 \mathrm{H}), 2.48-2.43(\mathrm{~m}, 1 \mathrm{H}), 2.16-1.82(\mathrm{~m}, 3 \mathrm{H}) ;{ }^{13} \mathrm{C} \mathrm{NMR}\left(\mathrm{CDCl}_{3}, 100 \mathrm{MHz}\right) 168.0,163.9,159.4$, 
134.2, 129.9, 129.3, 128.6, 127.5, 125.9, 114.3, 64.4, 58.3, 55.3, 47.3, 45.7, 29.5, 22.4; HRMS: calcd. for $\mathrm{C}_{21} \mathrm{H}_{23} \mathrm{~N}_{2} \mathrm{O}_{3}\left(\mathrm{M}^{+}+1\right)$ : 351.1709. Found 351.1709.

(3R,8aS)-2-(4-Methoxybenzyl)-3-methylhexahydropyrrolo[1,2-a] pyrazine-1,4-dione (44) A colorless oil was obtained in 76\% yield. ${ }^{1} \mathrm{H}$ NMR $\left(\mathrm{CDCl}_{3}, 400 \mathrm{MHz}\right) 7.19-7.13(\mathrm{~m}, 2 \mathrm{H}), 6.86$ $6.83(\mathrm{~m}, 2 \mathrm{H}), 5.30(\mathrm{~s}, 1 \mathrm{H}), 4.93(\mathrm{~d}, J 14.8 \mathrm{~Hz}, 1 \mathrm{H}), 4.17-4.11(\mathrm{~m}, 2 \mathrm{H}), 3.92-3.87(\mathrm{~m}, 1 \mathrm{H}), 3.79$ $(\mathrm{s}, 3 \mathrm{H}), 3.63-3.51(\mathrm{~m}, 2 \mathrm{H}), 2.48-2.44(\mathrm{~m}, 1 \mathrm{H}), 2.11-1.81(\mathrm{~m}, 3 \mathrm{H}), 1.35(\mathrm{~d}, J 6.8 \mathrm{~Hz}, 3 \mathrm{H}) ;{ }^{13} \mathrm{C}$ NMR ( $\left.\mathrm{CDCl}_{3}, 100 \mathrm{MHz}\right)$ 167.1, 166.9, 159.4, 129.8, 128.6, 114.3, 58.4, 57.5, 55.3, 47.1, 45.5, $29.4,22.5,16.7$. The spectral data of $\mathbf{4 4}$ were identical to those of the authentic materials reported previously. ${ }^{26}$

General procedure for the preparation of diastereomeric mixtures of $\alpha$-bromo tertiary amides. $N$-Alkyl-L-amino acid methyl ester (1.0 equiv), bromoacetic acid (1.0 equiv), DMAP (0.1 equiv) and DCC (1.1 equiv) were dissolved in $\mathrm{CH}_{2} \mathrm{Cl}_{2}$ and stirred at room temperature for 3 $\mathrm{h}$. The mixture was then worked up by extraction with EtOAc and the organic phase was dried over $\mathrm{MgSO}_{4}$. Filtration and concentration provided the crude product that was purified by column chromatography on silica gel. A 1:1 mixture of two diastereomers was obtained in 35$58 \%$ yields. No further attempts were made to optimize the yields. (Experimental procedures for the preparation of $1,12,13,14,15,32,33$ and 34 were described in previous publications. ${ }^{14}$ ) $\boldsymbol{N}$-Benzyl- $\boldsymbol{N}$-[ $\alpha$-bromo- $\alpha$-(4-chlorophenyl)acetyl]-(S)-leucine methyl ester (16) ${ }^{1} \mathrm{H}$ NMR $\left(\mathrm{CDCl}_{3}, 400 \mathrm{MHz}\right.$, two rotamers of two diastereomers) 7.97-7.17 (m, 9H), 5.42, $5.37(\mathrm{~s}, 1 \mathrm{H})$, 5.15-4.45 (m, 3H), 3.72, 3.68, 3.56, $3.50(\mathrm{~s}, 3 \mathrm{H}), 1.82(\mathrm{~m}, 1 \mathrm{H}), 1.66-1.52(\mathrm{~m}, 2 \mathrm{H}), 0.95-0.75(\mathrm{~m}$, $6 \mathrm{H}) ;{ }^{13} \mathrm{C} \mathrm{NMR}\left(\mathrm{CDCl}_{3}, 100 \mathrm{MHz}\right) 171.6,168.7,136.9,136.7,135.1,134.4,131.2,130.7,130.6$, $129.2,128.9,128.7,128.4,128.1,128.0,126.1,57.5,56.4,52.2,52.1,50.4,49.4,44.8,44.2$, 38.4, 38.2, 25.3, 25.1, 22.7, 22.4, 22.3; HRMS: calcd. for $\mathrm{C}_{22} \mathrm{H}_{26} \mathrm{BrClNO}_{3}\left(\mathrm{M}^{+}+1\right)$ 466.0785; found 466.0785 .

$\boldsymbol{N}$-Benzyl- $\boldsymbol{N}$-[ $\alpha$-bromo- $\alpha$-(4-bromophenyl)acetyl]-(S)-leucine methyl ester (17) ${ }^{1} \mathrm{H}$ NMR $\left(\mathrm{CDCl}_{3}, 400 \mathrm{MHz}\right.$, two rotamers of two diastereomers) 7.47-7.19 (m, 9H), 5.44, $5.42(\mathrm{~s}, 1 \mathrm{H})$, 5.04-4.50 (m, 3H), 3.71, 3.67, 3.63, $3.58(\mathrm{~s}, 3 \mathrm{H}), 1.90-1.82(\mathrm{~m}, 1 \mathrm{H}), 1.58-1.51(\mathrm{~m}, 2 \mathrm{H}), 0.94-$ $0.76(\mathrm{~m}, 6 \mathrm{H}) ;{ }^{13} \mathrm{C} \mathrm{NMR}\left(\mathrm{CDCl}_{3}, 100 \mathrm{MHz}\right) 171.5,171.4,168.6,168.4,136.9,136.7,136.5$, $134.8,134.6,132.2,131.9,131.8,131.7,131.3,131.0,130.9,130.3,130.1,129.8,129.2,128.9$, $128.6,128.4,128.1,128.0,127.7,127.2,126.4,126.3,126.2,126.1,123.5,123.4,59.1,57.5$, $57.4,56.8,56.2,52.2,52.1,51.7,50.3,49.6,44.8,44.3,42.6,38.4,38.3,38.2,25.3,25.2,25.1$, 24.9, 22.8, 22.7, 22.6, 22.4, 22.3, 22.2; HRMS: calcd. for $\mathrm{C}_{22} \mathrm{H}_{26} \mathrm{Br}_{2} \mathrm{NO}_{3}\left(\mathrm{M}^{+}+1\right)$ 510.0279; found 510.0279 .

$\boldsymbol{N}$-Benzyl- $\boldsymbol{N}$-( $\boldsymbol{\alpha}$-bromopropionyl)-(S)-leucine methyl ester (18) ${ }^{1} \mathrm{H}$ NMR $\left(\mathrm{CDCl}_{3}, 400 \mathrm{MHz}\right.$, two rotamers of two diastereomers) $7.40-7.19(\mathrm{~m}, 5 \mathrm{H}), 5.16-4.38(\mathrm{~m}, 4 \mathrm{H}), 3.72-3.41(\mathrm{~s}, 3 \mathrm{H})$, 1.95-1.25 (m, 6H), 0.93-0.75 (m, 6H); ${ }^{13} \mathrm{C} \mathrm{NMR}\left(\mathrm{CDCl}_{3}, 100 \mathrm{MHz}\right.$, two rotamers of two diastereomers) $171.7,171.5,170.7,170.6,137.0,136.6,129.0,128.9,128.4,127.8,127.7,127.3$, $126.2,126.1,57.6,55.4,52.3,51.9,50.8,48.8,39.1,39.0,38.5,37.9,25.3,24.9,24.8,22.9,22.5$, 22.3, 22.2, 21.4; HRMS: calcd. for $\mathrm{C}_{17} \mathrm{H}_{25} \mathrm{BrNO}_{3}\left(\mathrm{M}^{+}+1\right) 370.1018$; found 370.1010 . 
$\boldsymbol{N}$-Benzyl- $\boldsymbol{N}$-( $\boldsymbol{\alpha}$-bromobutanoyl)-(S)-leucine methyl ester (19) ${ }^{1} \mathrm{H}$ NMR $\left(\mathrm{CDCl}_{3}, 400 \mathrm{MHz}\right.$, two rotamers of two diastereomers) 7.40-7.22 (m, 5H), 5.13-4.08 (m, 4H), 3.72-3.49 $(\mathrm{s}, 3 \mathrm{H})$, 2.25-0.68 (m, $15 \mathrm{H}) ;{ }^{13} \mathrm{C} \mathrm{NMR}\left(\mathrm{CDCl}_{3}, 100 \mathrm{MHz}\right.$, two rotamers of two diastereomers) 171.6, $170.0,137.0,136.7,128.9,128.8,128.3,127.8,127.7,126.2,57.8,55.6,52.2,51.9,50.9,49.0$, 46.0, 45.6, 38.5, 37.9, 28.4, 28.3, 25.3, 24.9, 22.8, 22.5, 22.3, 22.2, 12.2; HRMS: calcd. for $\mathrm{C}_{18} \mathrm{H}_{27} \mathrm{BrNO}_{3}\left(\mathrm{M}^{+}+1\right) 384.1174$; found 384.1183 .

$\boldsymbol{N}$-( $\alpha$-Bromo- $\alpha$-phenylacetyl)- $\boldsymbol{N}$-(4-methoxybenzyl)-(S)-leucine methyl ester (28) ${ }^{1} \mathrm{H}$ NMR $\left(\mathrm{CDCl}_{3}, 400 \mathrm{MHz}\right.$, two rotamers of two diastereomers) 7.41-6.89 (m, 9H), 5.56, $5.52(\mathrm{~s}, 1 \mathrm{H})$, 5.02-4.43 (m, 3H), 3.82, 3.64, 3.57 (s, 3H), 1.90-1.83 (m, 1H), 1.64-1.49 (m, 2H), 0.93-0.77 (m, $6 \mathrm{H}) ;{ }^{13} \mathrm{C} \mathrm{NMR}\left(\mathrm{CDCl}_{3}, 100 \mathrm{MHz}\right) 171.6,168.7,168.2,159.3,136.0,135.9,129.1,129.0,128.7$, $128.6,128.5,128.4,127.6,127.5,114.4,113.7,57.2,56.7,55.4,52.1,52.0,49.3,46.7,46.2$, 38.3, 38.2, 25.2, 25.1, 22.7, 22.5, 22.3; HRMS: calcd. for $\mathrm{C}_{23} \mathrm{H}_{29} \mathrm{BrNO}_{4}\left(\mathrm{M}^{+}+1\right) 462.1280$; found 462.1280 .

$\boldsymbol{N}$-( $\alpha$-Bromo- $\alpha$-phenylacetyl)- $\mathrm{N}$-(4-methoxybenzyl)-(S)-alanine methyl ester (29) ${ }^{1} \mathrm{H}$ NMR $\left(\mathrm{CDCl}_{3}, 400 \mathrm{MHz}\right.$, two rotamers of two diastereomers) 7.46-6.89 (m, 9H), 5.62, 5.51, 5.27 (s, $1 \mathrm{H}), 4.74-4.46(\mathrm{~m}, 3 \mathrm{H}), 3.81,3.76,3.69,3.66(\mathrm{~s}, 6 \mathrm{H}), 1.41,1.37$ (d, J 7.2 Hz, 3H); ${ }^{13} \mathrm{C} \mathrm{NMR}$ $\left(\mathrm{CDCl}_{3}, 100 \mathrm{MHz}\right.$, two rotamers of two diastereomers) 171.6, 168.3, 167.9, 159.3, 136.0, 129.2, $129.1,128.7,128.6,128.5,128.1,127.6,127.5,114.5,114.4,55.4,55.2,55.1,52.3,50.3,50.0$, 47.8, 45.8, 14.5; HRMS: calcd. for $\mathrm{C}_{20} \mathrm{H}_{23} \mathrm{BrNO}_{4}\left(\mathrm{M}^{+}+1\right)$ 420.0810; found 420.0799.

$\boldsymbol{N}$-(a-Bromopropionyl)- $\boldsymbol{N}$-(4-methoxybenzyl)-(S)-alanine methyl ester (30) ${ }^{1} \mathrm{H} \mathrm{NMR}\left(\mathrm{CDCl}_{3}\right.$, $400 \mathrm{MHz}$, two rotamers of two diastereomers) 7.40-7.19 (m, 4H), 5.15-4.36 (m, 4H), 3.73, 3.58, 3.47 (s, 6H), 1.88, 1.77 (d, J 7.2 Hz, 3H), 0.93-0.74 (m, 3H); ${ }^{13} \mathrm{C} \mathrm{NMR} \mathrm{(CDCl}, 100 \mathrm{MHz}$, two rotamers of two diastereomers) $171.7,170.6,137.0,129.0,128.9,128.4,127.8,127.3,126.1$, 57.6, 52.3, 50.8, 39.0, 38.3, 37.9, 25.3, 22.9, 22.5, 22.3, 22.2, 21.4; HRMS: calcd. for $\mathrm{C}_{15} \mathrm{H}_{21} \mathrm{BrNO}_{4}\left(\mathrm{M}^{+}+1\right) 358.0654$; found 358.0654 .

$\boldsymbol{N}$-( $\boldsymbol{\alpha}$-Bromo- $\boldsymbol{\alpha}$-phenylacetyl)- $\boldsymbol{N}$-cinnamyl-(S)-leucine methyl ester (31) ${ }^{1} \mathrm{H} \mathrm{NMR}\left(\mathrm{CDCl}_{3}, 400\right.$ $\mathrm{MHz}$, two rotamers of two diastereomers) 7.54-7.26 (m, 10H), $6.54(\mathrm{~m}, 1 \mathrm{H}), 6.13(\mathrm{~m}, 1 \mathrm{H}), 5.80$, 5.78, $5.76(\mathrm{~s}, 1 \mathrm{H}), 5.14,4.98(\mathrm{~m}, 1 \mathrm{H}), 4.32-4.00(\mathrm{~m}, 2 \mathrm{H}), 3.68,3.62(\mathrm{~s}, 3 \mathrm{H}), 1.87-1.64(\mathrm{~m}, 3 \mathrm{H})$, 0.98-0.86 (m, 6H); ${ }^{13} \mathrm{C} \mathrm{NMR}\left(\mathrm{CDCl}_{3}, 100 \mathrm{MHz}\right.$, two rotamers of two diastereomers $) 171.8$, $168.6,168.4,136.2,136.0,135.8,132.5,129.1,128.8,128.7,128.4,128.2,126.5,125.1,56.7$, 56.1, 52.3, 52.1, 48.7, 48.1, 46.5, 38.0, 25.1, 25.0, 23.0, 22.8, 22.1, 22.0; HRMS: calcd. for $\mathrm{C}_{24} \mathrm{H}_{29} \mathrm{BrNO}_{3}\left(\mathrm{M}^{+}+1\right)$ 458.1331; found 458.1327 .

\section{Supplementary Material}

The NMR of new compounds. 


\section{Acknowledgements}

This work was supported by Basic Science Research Program through the National Research Foundation of Korea (NRF) funded by the Ministry of Education (2014R1A1A2057279).

\section{References}

1. Martins, M. B.; Carvalho, I. Tetrahedron 2007, 63, 9923. http://dx.doi.org/10.1016/j.tet.2007.04.105

2. Borthwick, A. D. Chem. Rev. 2012, 112, 3641. http://dx.doi.org/10.1021/cr200398y

3. Wang, H.; Usui, T.; Osada, H.; Ganesan, A. J. Med. Chem. 2000, 43, 1577. http://dx.doi.org/10.1021/jm9905662

4. Airaghi, F.; Fiorati, A.; Lesma, G.; Musolino, M.; Sacchetti, A.; Silvani, A. Beilstein J. Org. Chem. 2013, 9, 147.

http://dx.doi.org/10.3762/bjoc.9.17

5. Panzeri, S.; Zanella, S.; Arosio, D.; Vahdati, L.; Corso, A. D.; Pignataro, L.; Paolillo, M.; Schinelli, S.; Belvisi, L.; Gennari, C.; Piarulli, U. Chem. Eur. J. 2015, 21, 6265. http://dx.doi.org/10.1002/chem.201406567

6. Zhang, H.; Wang, Q.; Ning, X.; Hang, H.; Ma, J.; Yang, X.; Lu, X.; Zhang, J.; Li, Y.; Niu, C.; Song, H.; Wang, X.; Wang, P. G. J. Agric. Food Chem. 2015, 63, 3734. http://dx.doi.org/10.1021/jf506153t

7. Bianco, A.; Sonksen, C. P.; Roepstorff, P.; Briand, J.-P. J. Org. Chem. 2000, 65, 2179. http://dx.doi.org/10.1021/jo991818+

8. Veerman, J. J. N.; Bon, R. S.; Hue, B. T. B.; Girones, D.; Rutjes, F. P. J. T.; van Maarseveen, J. H.; Hiemstra, H. J. Org. Chem. 2003, 68, 4486. http://dx.doi.org/10.1021/jo0342572

9. Tullberg, M.; Grøtli, M.; Luthman, K. J. Org. Chem. 2007, 72, 195. http://dx.doi.org/10.1021/jo0619635

10. Santra, S.; Andreana, P. R. Org. Lett. 2007, 9, 5035. http://dx.doi.org/10.1021/o1702256t

11. Nikulnikov, M.; Shumsky, A.; Krasavin, M. Synthesis 2015, 15, 2527.

12. Lim, H. J.; Gallucci, J. C.; RajanBabu, T. V. Org. Lett. 2010, 12, 2162. http://dx.doi.org/10.1021/o1100663y

13. Jida, M.; Tourwé, D.; Ballet, S. RSC Adv. 2014, 4, 38159. http://dx.doi.org/10.1039/C4RA05981F

14. Baek, J.; Kang, S. Y.; Im, C.; Park, Y. S. Eur. J. Org. Chem. 2014, 2780. http://dx.doi.org/10.1002/ejoc.201301936 
15. The enantiomeric purity of $\mathbf{2}$ was confirmed to be $>99: 1$ er by chiral stationary phase HPLC (Chiralpak AD-H column) using racemic 2 as a standard.

16. The configurational stability of tetrasubstituted 2,5-diketopiperazine $\mathbf{2}$ under the reaction conditions was examined by the treatment of $2(85: 15 \mathrm{dr})$ with benzylamine, TBAI and DIEA in $\mathrm{CH}_{3} \mathrm{CN}$ for two days. No epimerization was detected by ${ }^{1} \mathrm{H}-\mathrm{NMR}$, thus ruling out the possibility of epimerization after replacement of $\mathrm{Br}$ with the nucleophile.

17. Nam, J.; Chang, J.-y.; Shin, E.-k.; Kim, H. J.; Kim, Y.; Jang, S.; Park, Y. S. Tetrahedron 2004, 60, 6311.

http://dx.doi.org/10.1016/j.tet.2004.05.094

18. Caddick, S.; Afonso, C. A. M.; Candeias, S. X.; Hitchcock, P. B.; Jenkins, K.; Murtagh, L.; Pardoe, D.; Santos A. G.; Treweeke, N. R.; Weaving, R. Tetrahedron 2001, 57, 6589. http://dx.doi.org/10.1016/S0040-4020(01)00550-6

19. Devine, P. N.; Foster, B. S.; Grabowski, E. J. J.; Reider, P. J. Heterocycles 2002, 58, 119. http://dx.doi.org/10.3987/COM-02-S(M)54

20. Valenrod, Y.; Myung, J.; Ben, R. N. Tetrahedron Lett. 2004, 45, 2545. http://dx.doi.org/10.1016/j.tetlet.2004.02.004

21. Bull, S. D.; Davies, S. G.; Garner, A. C.; O’Shea, M. D J. Chem. Soc., Perkin Trans. 1 2001, 3281.

22. Bull, S. D.; Davies, S. G.; Garner, Savory, A. G.; Snow, E. D. E. J.; Smith, A. D. Tetrahedron: Asymmetry 2004, 15, 3989.

http://dx.doi.org/10.1016/j.tetasy.2004.11.022

23. The treatment of $\mathbf{3 8}$ with CAN to remove both $p$-methoxybenzyl groups afforded cis-5methyl-3-phenyl-2,5-diketopiperazine.

24. Khimiuk, A. Y.; Korennykh, A. V.; van Langen, L. M.; van Rantwijk, F.; Sheldonb, R. A.; Svedas, V. K. Tetrahedron: Asymmetry 2003, 14, 3123. http://dx.doi.org/10.1016/j.tetasy.2003.08.011

25. The configuration of $\mathbf{3 9}$ and $\mathbf{4 4}$ was determined by comparing their NMR spectra with those of previously reported compounds.

26. Pichowicz, M.; Simpkins, N. S.; Blake, A. J.; Wilson, C. Tetrahedron 2008, 64, 3713. http://dx.doi.org/10.1016/j.tet.2008.02.020 